\title{
Simulation Of An Antihydrogen Gravity Experiment Utilizing Multiple Apertures
}

\author{
R. M. Hedlof and C. A. Ordonez \\ Department of Physics, University of North Texas, Denton, Texas 76203, USA
}

\begin{abstract}
An analytical model and a Monte Carlo simulation of an antihydrogen gravity experiment that would employ multiple apertures are presented. Such an experiment may be possible at the CERN Antiproton Decelerator facility. The model was developed with the primary goal of reducing the experimental run time necessary to determine the direction of free fall acceleration for antimatter in the gravitational field of the Earth. The experiment would confine cryogenic antihydrogen plasma for producing antihydrogen (e.g., by three-body recombination). A cylindrical drift tube would have a horizontal axis of symmetry, with a series of coaxial apertures positioned on one or both sides of the region for antihydrogen production. The experiment would employ a detector capable of distinguishing between cosmic rays and antihydrogen annihilations. The distribution of annihilations on the drift tube would be azimuthally asymmetric for a short distance beyond each aperture within a shadow region depending on the direction of the gravitational acceleration of antimatter. The analytical model is used to determine the probability that an antiatom would annihilate within one of the shadow regions for specified experimental parameters.
\end{abstract}

Keywords: Antihydrogen, Gravity, CERN AD.

PACS: 52.65.Pp,04.80.Cc,52.27.Jt

\section{INTRODUCTION}

There has never been a measurement that indicates the direction of acceleration of antimatter in the presence of Earth's gravitational field. Past attempts using charged antimatter particles have been thwarted by stray electromagnetic fields. Antihydrogen is the most basic form of neutral antimatter, and is the counterpart of the most extensively studied system of normal matter, hydrogen. An experiment that would implement multiple apertures positioned on either side of an antihydrogen production region may be feasible. Ultimately, comparisons between the properties of hydrogen and antihydrogen may provide tests of the Charge, Parity, and Time theorem as well as gravity symmetries [1-7].

An analytical model and a simulation of an antihydrogen gravity experiment are presented here. The model and simulation were developed with the primary goal of determining the direction of free fall acceleration of antimatter for the first time, with the minimization of experimental run time necessary to achieve this goal. The experiment would require the use of a detection system capable of distinguishing between antihydrogen annihilations and cosmic rays. Such a detection system is currently used for antihydrogen research by the ALPHA collaboration [8].
A conceptual illustration of a possible experimental configuration utilizing three apertures on only one side of the antihydrogen production region is shown in Fig. 1, and Fig. 2 depicts a single arbitrary aperture $A_{i}$. The

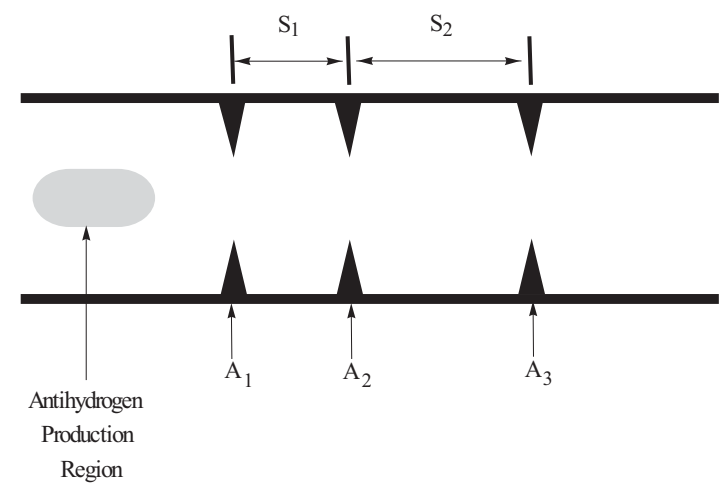

FIGURE 1. A conceptual illustration of an experimental configuration using three apertures $A_{1}, A_{2}$, and $A_{3}$ positioned on only one side of the antihydrogen production region. Each successive aperture is positioned at the end of the previous aperture's "shadow region," which is defined in Fig. 2.

two straight line trajectories in Fig. 2 are representative of antiatoms with sufficiently high energies that their trajectories are not significantly affected by gravity. These trajectories, passing

Application of Accelerators in Research and Industry

AIP Conf. Proc. 1525, 102-105 (2013); doi: 10.1063/1.4802299

(C) 2013 AIP Publishing LLC 978-0-7354-1148-7/ $\$ 30.00$ 
infinitesimally close to the selected aperture, intersect the drift tube a distance $\mathrm{z}_{\mathrm{i}}$ from the origin. The shadow region $S_{i}$, for the $i$ th aperture, is defined as the region on the inner surface of the drift tube from the aperture to the point at which these trajectories intersect the drift tube. Aside from the apertures adjacent to the antihydrogen production region, each consecutive aperture is located at the edge of the shadow region associated with the previous aperture closest to the origin. If it is assumed that all effects other than gravity that could otherwise alter the trajectory of an antiatom are axisymmetric, then the distribution of annihilations within the shadow region would be azimuthally asymmetric, depending on the direction of gravitational acceleration.

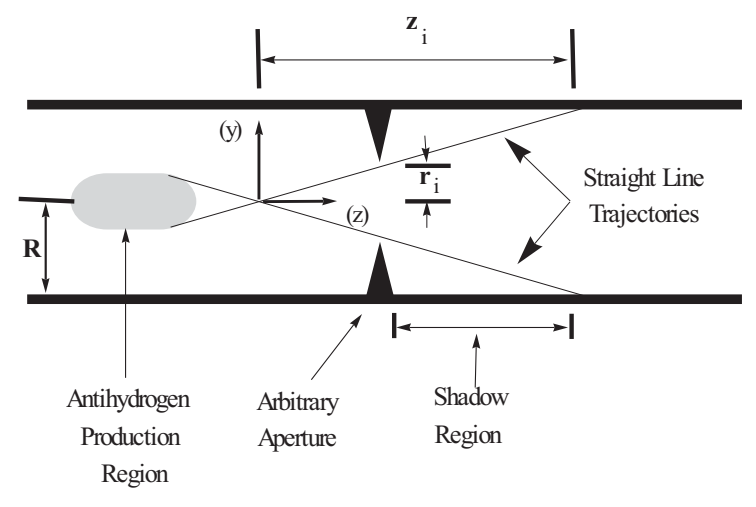

FIGURE 2. A conceptual illustration depicting relevant parameters corresponding to each aperture.

\section{ANALYTICAL MODEL}

To develop an analytical model that can be used to determine the probability that an antiatom will annihilate within the shadow region of the $i$ th aperture, the motion of an antiatom is first restricted to the $y-z$ plane. Furthermore all antiatoms are considered to be produced from a point source located at the origin and are subject to a gravitational acceleration equal in magnitude and opposite in direction to that of ordinary matter. A Cartesian coordinate system with associated unit vectors $(\mathbf{i}, \mathbf{j}, \mathbf{k})$ is defined with the $\mathrm{z}$-axis oriented along the axis of symmetry of the drift tube, and the positive y-axis directed vertically away from the center of the Earth. The acceleration due to gravity for each antiatom is thus $\boldsymbol{g}=\boldsymbol{g} \boldsymbol{j}$, where $\mathrm{g}$ is the magnitude of the gravitational acceleration matter experiences near the surface of the Earth. The equations of motion in the plane are given by $y(t)=v_{y_{0}} t+\frac{1}{2} g t^{2}$ and $z(t)=v_{z 0} t$. Here $\mathrm{y}$ and $\mathrm{z}$ are the vertical and horizontal displacements of an antiatom at any given time $\mathrm{t}$, and the velocity components may be written as $v_{y_{0}}=v_{0} \sin \theta_{0}$ and $v_{z 0}=v_{0} \cos \theta_{0}$. The equations of motion may be combined by eliminating time to yield $y=z \tan \theta_{0}+g \frac{z^{2}}{v_{0}^{2} \cos ^{2} \theta_{0}}$.

The initial velocity of an antiatom may be written in terms of its initial kinetic energy as $v_{0}=\sqrt{2 \frac{k_{0}}{m}}$. There exist certain limits on the values which the initial kinetic energy and angle of inclination may take in order for the antiatom to annihilate on the top of the drift tube within the shadow region. A lower limit on the kinetic energy is associated with a trajectory that passes infinitesimally close to the edge of the aperture with radius $r_{i}$. At the time the antiatom reaches the aperture $A_{i}$, the $\mathrm{y}$ and $\mathrm{z}$ coordinates are given by $y=r_{i}$ and $z=r_{i} z_{i} / R$, where $z_{i}$ is the $\mathrm{z}$ coordinate corresponding to the edge of the $i$ th shadow region. Substitution and rearrangement yields the minimum kinetic energy necessary to reach the $i$ th shadow region

$$
K_{0, \min }=\frac{m g z_{i}^{2}}{\left[4 R / r_{i}\left(R-z_{i} \tan \theta_{0}\right) \cos ^{2} \theta_{0}\right]} .
$$

The upper limit on the kinetic energy is associated with a trajectory that intersects the drift tube infinitesimally close to the edge of the $i$ th shadow region. In the ALPHA experiment the axial resolution of the detector implemented is approximately $\zeta=5 \mathrm{~mm}$. We only consider antiatoms which annihilate on the drift tube a distance greater than $5 \mathrm{~mm}$ from the edge of the shadow region. The coordinates at the time an antiatom with such a trajectory reaches the drift tube are given by $y=R$ and $z=z_{i}-\zeta$. The maximum initial kinetic energy possible for an antiatom to have a trajectory that would intersect the drift tube within the restricted portion of the $i$ th shadow region is given by

$$
K_{0, \max }=\frac{m g\left(z_{i}-\zeta\right)^{2}}{\left[4\left(R-\left(z_{i}-\zeta\right) \tan \theta_{0}\right) \cos ^{2} \theta_{0}\right]} .
$$

A lower bound on the initial angle of inclination is taken to be

$$
\theta_{0, \min }=0
$$

so that it may later be used as a spherical coordinate in velocity space. A trajectory that passes infinitesimally close to the upper edge of an aperture and also annihilates infinitesimally close to $z=z_{i}-\zeta$ corresponds to the maximum initial angle of inclination. Setting $K_{0, \min }$ equal to $K_{0, \max }$, and solving for $\theta_{0}$ provides an expression for the maximum angle of inclination: 


$$
\theta_{0, \max }=\arctan \left[R\left(\frac{1}{z_{i}}+\frac{1}{z_{i}-\zeta}-\frac{1}{z_{i}-\frac{\zeta}{1-\frac{r_{i}}{R}}}\right)\right] \text {. }
$$

TABLE 1. Summary of results for single-aperture Monte Carlo simulations and analytical calculations. Parameters that are the same for all results are $R=0.0223 \mathrm{~m}, r_{i}=\frac{R}{2}=0.01115 \mathrm{~m}, \zeta=0.005 \mathrm{~m}$, and $N=3.0 \times 10^{8}$. The probability for an antiatom to annihilate within a restricted portion, $z_{a 1}<z<z_{1}-\bar{\zeta}$, of the shadow region is shown in the first and second columns. The first column is the probability according to the analytical model. The second is the probability according to the Monte Carlo simulations. The symbols $N$ and $N_{\zeta}$ denote the total number of atoms and the number of annihilations within the restricted portion of the shadow region respectively.

\begin{tabular}{|c|c|c|c|c|c|}
\hline$p=\frac{1}{3} P\left(\times 10^{-8}\right)$ & $\frac{S N_{Z^{3}}}{N} \pm \frac{\sigma}{N}\left(\times 10^{-8}\right)$ & $<N_{\zeta}> \pm \sigma$ & $\mathbf{T}(\mathrm{K})$ & $Z_{1}(m)$ & $N_{s}$ \\
\hline .51 & $.50 \pm 0.33$ & $1.5 \pm 1.0$ & 4 & 0.2 & 930,000 \\
\hline 18 & $21 \pm 3.3$ & $63 \pm 10$ & 4 & 2.7 & 5,200 \\
\hline 2.7 & $2.7 \pm 0.47$ & $8.1 \pm 1.4$ & 4 & 0.5 & 150,000 \\
\hline .088 & $0.067 \pm 0.14$ & $0.20 \pm 0.41$ & 40 & 0.5 & 150,000 \\
\hline 79 & $83 \pm 6.7$ & $250 \pm 20$ & 0.4 & 0.5 & 150,000 \\
\hline
\end{tabular}

Under the conditions imposed thus far, it is not possible for annihilations to occur within any of the shadow regions on the lower half of the drift tube. Alternatively, if antimatter experiences a gravitational acceleration equal to matter, it is impossible for annihilations to occur within any of the shadow regions on the upper half of the drift tube. A maximal number of annihilations within the $i$ th shadow region correspond to antiatoms born with initial kinetic energies and initial angles of inclination satisfying $K_{0, \min } \leq K_{0} \leq K_{0, \max }$ and $\theta_{0, \min } \leq \theta_{0} \leq \theta_{0, \max }$. The probability for an antiatom to satisfy these conditions is evaluated by assuming that the antiatoms follow a Maxwellian velocity distribution. This assumption is consistent with the production of antihydrogen within a thermalized antihydrogen plasma that is magnetically confined, provided the difference between the positron and antiproton densities is such that the $\mathbf{E} \times \mathbf{B}$ drift speed is much smaller than the antiproton thermal speed throughout the plasma. After a change of variables in the velocity space probability density by the relation $v_{0}=\sqrt{2 K_{0} / m}$, the twodimensional probability of an antiatom to annihilate within the $i$ th shadow region is given by evaluating the definite integral

$P_{i}=\iint \frac{\sin \theta_{0}}{\sqrt{\pi\left(k_{B} T\right)^{3}}} \sqrt{K_{0}} \exp \left(-\frac{K_{0}}{k_{B} T}\right) d K_{0} d \theta_{0}$

in which the limits of integration run from $K_{0, \min }$ to $K_{0, \max }$ and $\theta_{0, \min }$ to $\theta_{0, \max }$ respectively.

A comparison shown in Table 1 with the probability evaluated using three-dimensional Monte Carlo simulations reveals that the probability in three dimensions for an antiatom to annihilate within a restricted portion of the shadow region, $z_{a i} \leq z \leq z_{i}-\zeta$, of the $i$ th shadow region is given by $p_{i}=\frac{1}{3} P_{i}$, where $P_{i}$ is given by Eq. (5). Thus the total probability for an antiatom to annihilate within the restricted portion of $n$ shadow regions is given by,

$$
p=\sum_{i=1}^{n} p_{i}=\frac{1}{3} \sum_{i=1}^{n} P_{i}
$$

It should be noted that similar simulations for a single aperture also show that the probability of annihilations within each of the shadow regions is decreased by approximately $40 \%$ if a finite sized antihydrogen plasma is considered as opposed to the point source value for parameters similar to those reported by existing antihydrogen collaborations [9].

The results using the analytical model are shown in Table 2. The value for $z_{n}$ is assigned a value of $0.5 \mathrm{~m}$ corresponding to the extent of the uniform magnetic field within the ALPHA apparatus [7]. The temperature of the antihydrogen plasma is considered to be $4 \mathrm{~K}$, as the ATRAP collaboration reported the cooling of an antiproton plasma to a temperature of 3.5 $\mathrm{K}$ [10]. Each of the apertures is defined to have a radius of $r_{1}=R / 2$. The number of apertures is varied from 1 to 4, and the total probability for an antiatom to annihilate in the restricted portion of one of the regions is evaluated using Eqs. (5) and (6). The results indicate that implementation of multiple apertures would increase the probability for azimuthally asymmetric annihilations on the drift tube, therefore decreasing the experimental run time to determine the direction of free fall acceleration for antimatter.

\begin{tabular}{|c|c|}
\hline$n$ & $p\left(\times 10^{-8}\right)$ \\
\hline 1 & 2.7 \\
\hline 2 & 3.5 \\
\hline 3 & 3.7 \\
\hline 4 & 3.8 \\
\hline
\end{tabular}

TABLE 2. Summary of Analytical Results. 


\section{CONCLUSION}

A study of an antihydrogen gravity experiment using multiple apertures has been presented. An analytical model has been developed to give the probability for an antiatom to annihilate within any of the shadow regions for such an experiment. The results indicate that there will be an increase in the total number of annihilations within the shadow regions, thus reducing the experimental run time necessary to give an indication of the direction of free fall acceleration of antimatter within the gravitational field of the Earth.

\section{ACKNOWLEDGEMENTS}

This material is based upon work supported by the epartment of Energy under rant o. E- 0206ER 54883 and by the ational Science oundation under rant o. PH -1202428.

\section{REFERENCES}

1. . Robicheaux, Journal of Physics B: Atomic, Molecular and Optical Physics 41, 192001 (2008).

2. . abrielse, Advances in Atomic, Molecular, and Optical Physics 50, 155 (2005).

3. . abrisa et al., Nuclear Physics A 834, 751c (2010).

4. M. Charlton et al., Physics Reports 241, 65 (1994).

5. M. H. Holzscheiter, M. Charlton, and M. M. ieto, Physics Reports 402, 1 (2004).

6. C. M. Surko and R. . reaves, Physics of Plasmas 11, 2333 (2004).

7. . Madsen, Philosophical Transactions of the Royal Society A 368, 3671 (2010).

8. . . Andresen et al., Nature Physics 7, 558 (2011).

9. C. A. rdonez and R. M. Hedlof, AIP Advances 2, 012176 (2012).

10. . abrielse et al., Physical Review Letters 106, 073002 (2011). 